PROCEEDINGS OF THE

AMERICAN MATHEMATICAL SOCIETY

Volume 129, Number 2, Pages 459-465

S 0002-9939(00)05563-5

Article electronically published on August 28, 2000

\title{
PRODUCTS OF ROOTS OF THE IDENTITY
}

\author{
M. HLADNIK, M. OMLADIČ, AND H. RADJAVI \\ (Communicated by David R. Larson)
}

\begin{abstract}
It is proved that every invertible bounded linear operator on a complex infinite-dimensional Hilbert space is a product of five $n$-th roots of the identity for every $n>2$. For invertible normal operators four factors suffice in general.
\end{abstract}

\section{INTRODUCTION}

This is a contribution to the study of groups generated by those operators on a complex Hilbert space that are $n$-th roots of the identity operator for a fixed integer $n$. In other words, we are interested in products of operators of finite order whose orders divide $n$. Products of involutions, i.e., the case $n=2$, have been studied in both finite and infinite dimensions. In the finite-dimensional case, for example, this group is exactly the set of operators with determinant \pm 1 ; furthermore, every operator of the right determinant is the product of at most four involutions [4]. In infinite dimensions, the group of unitaries is generated by unitary involutions and four factors suffice to express every unitary operator [5]; the group of all invertible operators is generated by involutions and seven factors suffice [6]. Characterizations of products of two involutions have been given in [2, 7]. A more recent study in finite dimensions [3] concerns groups generated by elements of prime order.

We prove in this paper that $n$-th roots of the identity generate the group of all invertible operators and that five factors suffice if $n>2$. For normal operators we obtain sharper results which are intimately connected to the structure of group commutators given by Brown and Pearcy in [1] we make substantial use of their results.

\section{MAIN RESUlts}

In what follows all operators act on an infinite-dimensional separable Hilbert space and all results are given only in this setting. The necessary adjustments to the nonseparable case will be obvious to the reader.

Definition 1. Let $n$ be a positive integer. A bounded linear operator $X$ is called an $n$-th root (of the identity) if $X^{n}=I$, the identity operator.

Received by the editors September 1, 1998 and, in revised form, April 20, 1999.

2000 Mathematics Subject Classification. Primary 47A65; Secondary 47B47, 47D03.

Key words and phrases. Invertible operators, normal operators, compact operators, roots of the identity, group commutators.

This work was supported in part by the Ministry of Science and Technology of Slovenia and by the NSERC of Canada. 
Lemma 1. Let $T$ be of the form $T=A_{1} \oplus A_{2} \oplus \ldots \oplus A_{n}$ where the product $A_{1} A_{2} \ldots A_{n}$ is a group commutator. Then $T$ is a a product of three $n$-th roots for any given $n>2$.

Proof. Write the product $A_{1} A_{2} \ldots A_{n}$ as a commutator $U V U^{-1} V^{-1}$. Let $w$ be the $n \times n$ circulant matrix that sends the first basis vector to the last, the second to the first, the third to the second, etc. Let $W$ be the tensor product of $w$ with the identity operator $I$, i.e., $W=w \otimes I$. Define

$$
K_{1}=\left(U^{-1} A_{1} A_{2} \oplus A_{2}^{-1} A_{1}^{-1} U V A_{1} \oplus I \oplus \ldots \oplus I \oplus A_{1}^{-1} V^{-1}\right) W
$$

and

$$
K_{2}=\left(V A_{1} A_{2} \oplus A_{2}^{-1} A_{1}^{-1} U A_{1} \oplus A_{1}^{-1} V^{-1} U^{-1} A_{1} A_{2} A_{3} \oplus A_{4} \oplus \ldots \oplus A_{n-1} \oplus A_{n}\right) W .
$$

Then $K_{1}^{n}=K_{2}^{n}=I$ and $A_{1} \oplus A_{3} \oplus A_{4} \oplus \ldots \oplus A_{n} \oplus A_{2}=K_{1} K_{2} W^{-2}$. Since $T$ is block permutationally similar to $A_{1} \oplus A_{3} \oplus A_{4} \oplus \ldots \oplus A_{n} \oplus A_{2}$, we are done.

Remark 1. It is clear from the proof that at least one of the $n$-th roots, namely $W^{-2}$, is unitary and that for unitary $A_{i}, i=1,2, \ldots, n$, all $n$-th roots are unitary.

Note that any normal operator $N$ on an infinite-dimensional Hilbert space can be split into the direct sum of two normal operators acting on infinite-dimensional subspaces. (If $\sigma(N)$ is infinite, use the spectral projections corresponding to two disjoint infinite Borel subsets; otherwise split at least one infinite-dimensional eigenspace of $N$.) Consequently, every normal $N$ on an infinite-dimensional Hilbert space can be split into the direct sum of arbitrarily many normal operators acting on infinite-dimensional subspaces.

In the sequel we use this fact repeatedly in proving that a normal operator $N$ is a product of three $n$-th roots. In order to do this we write $N$ (in a specific way) as a direct sum of normals acting on infinite-dimensional subspaces, e.g.

$$
N=N_{1} \oplus N_{2} \oplus N_{3} \oplus \ldots \oplus N_{n-1} \oplus N_{n}
$$

(Note that the order of summands in this sum is not important since with the change of order we get a unitarily equivalent operator and this does not affect the representation of $N$ as a product of $n$-th roots.) Then we show that the product $T=N_{1} N_{2} N_{3} \ldots N_{n-1} N_{n}$ is a group commutator using theorems in 1 and Lemma 1 yields the result.

A demonstration of a technique we use is the following easily obtained result:

Proposition 1. If an invertible normal operator $N$ has a unitary direct summand acting on an infinite-dimensional subspace, then $N$ is a product of three $n$-th roots.

Proof. Write $N=N_{1} \oplus U_{2} \oplus U_{3} \oplus \ldots \oplus U_{2 n}$ with $N_{1}$ normal and $U_{i}$ unitary, and let

$$
T=\left(N_{1} \oplus U_{2}\right)\left(U_{3} \oplus U_{4}\right) \ldots\left(U_{2 n-1} \oplus U_{2 n}\right)=\left(N_{1} U_{3} \ldots U_{2 n-1}\right) \oplus\left(U_{2} U_{4} \ldots U_{2 n}\right) .
$$

Since the second direct summand here is unitary and acts on an infinite-dimensional subspace, we can use Theorem 2 of [1] which asserts that an invertible operator with an infinite-dimensional unitary direct summand is a group commutator. Hence, $T$ is a group commutator and the proof is finished using Lemma 1. 
Corollary 1. Every unitary operator on an infinite-dimensional Hilbert space is a product of three unitary $n$-th roots where $n>2$.

Remark 2. This result is best possible as the counterexample $U=\alpha I$ with $|\alpha|=1$ and $\alpha^{n} \neq 1$, shows: If $\alpha I=X Y$ with $X^{n}=Y^{n}=I$, then $X$ and $Y$ commute and we have $\alpha^{n} I=X^{n} Y^{n}=I$ which is impossible. Also, (for $n=2$ ) it is not possible to have $\alpha I=X Y Z$ with $X^{2}=Y^{2}=Z^{2}=I$ if $\alpha^{2} \neq \pm 1$ since otherwise we would have $\alpha Z=X Y$ and $\alpha^{-1} Z=Y X \sim X Y=\alpha Z$. Hence, $\alpha^{2} Z$ would be similar to $Z$ which is impossible unless $\alpha^{2}=1$ or $\alpha^{2}=-1$. Note, however, that every unitary operator is the product of four involutions (see [5]).

Denote the unit circle by $\mathbf{T}$ and the essential spectrum of an operator $T$ by $\sigma_{\text {ess }}(T)$.

Proposition 2. Let $N$ be an invertible normal operator and let $\alpha, \beta \in \sigma_{\text {ess }}(N)$ with $|\alpha| \neq|\beta|$. Then $N$ is a product of three $n$-th roots for any $n>2$ and at least one of the factors is unitary.

Proof. Let $|\alpha|<|\beta|$ and choose $\epsilon<(|\beta|-|\alpha|) / 2$. Then $N$ can be decomposed into the direct sum $N=N_{0} \oplus N^{\alpha} \oplus N^{\beta}$ where $\sigma\left(N_{0}\right) \subset\{z \in \mathbb{C} ;|\alpha|+\epsilon \leq|z| \leq|\beta|-\epsilon\}$, $\sigma\left(N^{\alpha}\right) \subset\{z \in \mathbb{C} ;|z| \leq|\alpha|+\epsilon\}$ and $\sigma\left(N^{\beta}\right) \subset\{z \in \mathbb{C} ;|z| \geq|\beta|-\epsilon\}$. The operators $N^{\alpha}$ and $N^{\beta}$ can be further split into the direct sum of arbitrarily many normal operators acting on infinite-dimensional subspaces and with their essential spectra including $\alpha$ and $\beta$, respectively. So, let

$$
N=N_{1}^{\alpha} \oplus N_{2}^{\alpha} \oplus \ldots \oplus N_{2 n}^{\alpha} \oplus N_{1}^{\beta} \oplus N_{2}^{\beta} \oplus \ldots \oplus N_{2 n}^{\beta}
$$

with $N_{0}$ incorporated into $N_{1}^{\beta}$. Define

$$
T_{1}=\prod_{k=1}^{n}\left(N_{2 k-1}^{\alpha} \oplus N_{2 k-1}^{\beta}\right), T_{2}=\prod_{k=1}^{n}\left(N_{2 k}^{\alpha} \oplus N_{2 k}^{\beta}\right) .
$$

We show that $T_{1}$ and $T_{2}$ are not essentially scalar. Suppose that $T_{1}$ is of the form $T_{1}=\lambda I+K, K$ compact. Then so are $\prod_{k=1}^{n} N_{2 k-1}^{\alpha}$ and $\prod_{k=1}^{n} N_{2 k-1}^{\beta}$. Take the essential norms on both sides to see that $|\lambda| \leq(|\alpha|+\epsilon)^{n}$ and $|\lambda| \geq(|\alpha|+\epsilon)(|\beta|-\epsilon)^{n-1}$ at the same time which is clearly impossible. Hence, $T_{1}$ is not of the above form and the same applies to the operator $T_{2}$. Therefore, $T=T_{1} \oplus T_{2}$ is a group commutator by Theorem 4 of [1] and $N$ is a product of three $n$-th roots (with at least one unitary) by Lemma 1 .

Proposition 3. Let $N$ be an invertible normal operator with $\sigma_{e s s}(N) \subset r \boldsymbol{T}$ for an $r>0$. Suppose $N$ has a direct summand of the form $\alpha I+K$ with $K$ compact and $\alpha \in \sigma_{\text {ess }}(N)$. If $r=1$ or if there is at least one point $\beta \in \sigma_{\text {ess }}(N)$ different from $\alpha$, then the operator $N$ is a product of three $n$-th roots for any $n>2$ at least one of which is unitary.

Proof. If $r=1$, we may assume that there is no unitary direct summand in $N$ since otherwise we can use Proposition 1. If in this case $\alpha$ is the only point in $\sigma_{\text {ess }}(N)$, then $N=\alpha I+K$ with $K$ compact and $|\alpha|=1$. By a further splitting $N=\left(\alpha I+K_{1}\right) \oplus\left(\alpha I+K_{2}\right) \oplus \ldots \oplus\left(\alpha I+K_{n}\right)$ we get the product of these summands of the form $T=\alpha^{n} I+K^{\prime}$ with $K^{\prime}$ compact, which is a group commutator by Theorem 3 of [1]. So, we are done in the usual way using Lemma 1. 
In the remainder of the proof we can therefore assume that there exists a point $\beta \neq \alpha$ in the essential spectrum $\sigma_{\text {ess }}(N)$. Decompose

$$
N=N_{1} \oplus\left(\alpha I+K_{2}\right) \oplus N_{2} \oplus\left(\alpha I+K_{4}\right) \oplus \ldots \oplus\left(\alpha I+K_{4 n-1}\right) \oplus\left(\alpha I+K_{4 n}\right)
$$

such that $\beta \in \sigma_{\text {ess }}\left(N_{1}\right) \cap \sigma_{\text {ess }}\left(N_{2}\right)$ and write

$$
\begin{aligned}
T_{1}= & N_{1}\left(\alpha I+K_{5}\right)\left(\alpha I+K_{9}\right) \ldots\left(\alpha I+K_{4 n-3}\right) \\
& \oplus\left(\alpha I+K_{2}\right)\left(\alpha I+K_{6}\right)\left(\alpha I+K_{10}\right) \ldots\left(\alpha I+K_{4 n-2}\right) \\
= & \left(\alpha^{n-1} N_{1}+K_{1}^{\prime}\right) \oplus\left(\alpha^{n} I+K_{1}^{\prime \prime}\right), \\
T_{2}= & N_{2}\left(\alpha I+K_{7}\right)\left(\alpha I+K_{11}\right) \ldots\left(\alpha I+K_{4 n-1}\right) \\
& \oplus\left(\alpha I+K_{4}\right)\left(\alpha I+K_{8}\right)\left(\alpha I+K_{12}\right) \ldots\left(\alpha I+K_{4 n}\right) \\
= & \left(\alpha^{n-1} N_{2}+K_{2}^{\prime}\right) \oplus\left(\alpha^{n} I+K_{2}^{\prime \prime}\right)
\end{aligned}
$$

with $N_{1}, N_{2}$ normal and $K_{2}, K_{4}, K_{5}, \ldots, K_{4 n}, K_{1}^{\prime}, K_{1}^{\prime \prime}, K_{2}^{\prime}, K_{2}^{\prime \prime}$ compact. Then neither $T_{1}$ nor $T_{2}$ can be of the form $\lambda I+K, K$ compact, and hence, $T=T_{1} \oplus T_{2}$ is a group commutator by Theorem 4 of [1]. The proof can be now completed by the use of Lemma 1.

Remark 3. Note that the condition that $N$ has a direct summand of the form $\alpha I+K$ with $K$ compact and $\alpha \in \sigma_{e s s}(N)$ is fulfilled if $\alpha$ is an isolated point in $\sigma_{\text {ess }}(N)$ or $\alpha$ is a limit point of a sequence of isolated eigenvalues of $N$ with finite multiplicities. Note also that for $r=1$ this condition is not necessary since if it is not fulfilled we can use Proposition 1 to get the same result. Hence, every invertible normal $N$ with $\sigma_{\text {ess }}(N) \subset \mathbf{T}$ is a product of three $n$-th roots.

Proposition 4. The invertible normal operator $N=\alpha I+K$ with $|\alpha| \neq 1$ and $K$ compact, acting on an infinite-dimensional Hilbert space, is a product of four $n$-th roots for any $n>2$ and it is not a product of three $n$-th roots for $n=3$.

Proof. Let $\omega$ be a complex $n$-th root of unity. Decompose $N=\alpha I+K$ into the direct sum of $n$ operators $\alpha I+K_{i}, i=0,1,2, \ldots, n-1$, on infinite dimensional subspaces and write $N_{1}=I \oplus \omega^{-1} I \oplus \omega^{-2} I \oplus \ldots \oplus \omega^{-n+1} I$ and $N_{2}=\left(\alpha I+K_{0}\right) \oplus$ $\omega\left(\alpha I+K_{1}\right) \oplus \omega^{2}\left(\alpha I+K_{2}\right) \oplus \ldots \oplus \omega^{n-1}\left(\alpha I+K_{n-1}\right)$. Then $N=N_{1} N_{2}$ where the first factor is a (unitary) $n$-th root and the second factor fulfills the conditions of Proposition 3 since $\alpha$ is an isolated point in $\sigma_{e s s}\left(N_{2}\right)$. Hence, $N_{2}$ is a product of three $n$-th roots and $N$ is a product of four $n$-th roots for any $n>2$.

This result is the best possible in the case $n=3$, i.e., only three factors do not suffice for a normal $N=\alpha I+K, K$ compact, if $|\alpha| \neq 1$. To see this write $\alpha I+K=X Y Z$ with $X^{3}=Y^{3}=Z^{3}=I$ or $\alpha Z^{-1}+K Z^{-1}=X Y$. It follows that $\alpha^{3} I+K^{\prime}=(X Y)^{3}$ where $K^{\prime}$ is another compact operator. By the easily verifiable formula

$$
(X Y)^{3}=\left[[X, Y], Y X^{2}\right] Y X^{3} Y^{2},
$$

valid for every pair of operators $X, Y$, where the brackets $[X, Y]$ denote group commutators, we see that $(X Y)^{3}$ is always a group commutator for $X^{3}=I$ and $Y^{3}=I$. Since $|\alpha| \neq 1$, this is not possible for $\alpha^{3} I+K^{\prime}$ by Theorem 1 of [1].

Remark 4. Also, $\alpha I$ with $|\alpha| \neq 1$ cannot be a product of only three involutions as we saw in Remark 2. However, it is unclear whether such an operator is always a product of four involutions or whether it can be a product of only three $n$-th roots for $n>3$. 
Since for a normal operator every nonisolated spectral point belongs to its essential spectrum, it remains to consider invertible normal operators of the form $N=r U \oplus K$ where $r>0, r \neq 1, K$ is acting on a finite-dimensional subspace, and $U$ is unitary with more than one nonisolated point in its spectrum.

Proposition 5. Let $U$ be unitary without isolated points in its spectrum and let the invertible $N$ be of the form $N=r U \oplus K, 1 \neq r>0, K$ normal compact. Then for any $n>2$ the operator $N$ is a product of three $n$-th roots at least one of which is unitary.

Proof. Take any $\alpha \in \sigma(U)$. The idea is to decompose $U=U_{1} \oplus U_{2} \oplus \ldots \oplus U_{n} \oplus V_{1} \oplus$ $V_{2} \oplus \ldots \oplus V_{n}$ into infinite-dimensional unitaries in such a way that

$$
\left\|U_{i}-\alpha I\right\|<\frac{1}{2 n} \operatorname{diam} \sigma\left(U_{n}\right),\left\|V_{i}-\alpha I\right\|<\frac{1}{2 n} \operatorname{diam} \sigma\left(V_{n}\right)
$$

for $i=1,2, \ldots, n-1$. Then from

$$
\begin{aligned}
& U_{1} U_{2} U_{3} \ldots U_{n-1}-\alpha^{n-1} I \\
& =U_{1} U_{2} U_{3} \ldots U_{n-1}-\alpha U_{2} U_{3} \ldots U_{n-1}+\alpha U_{2} U_{3} \ldots U_{n-1}-\alpha^{2} U_{3} \ldots U_{n-1} \\
& \quad+\ldots+\alpha^{n-2} U_{n-1}-\alpha^{n-1} I
\end{aligned}
$$

we get

$$
\begin{aligned}
\left\|U_{1} U_{2} U_{3} \ldots U_{n-1}-\alpha^{n-1} I\right\| & \leq\left\|U_{1}-\alpha I\right\|+\left\|U_{2}-\alpha I\right\|+\ldots+\left\|U_{n-1}-\alpha I\right\| \\
& <\operatorname{diam} \sigma\left(U_{n}\right) / 2
\end{aligned}
$$

and, similarly, $\left\|V_{1} V_{2} V_{3} \ldots V_{n-1}-\alpha^{n-1} I\right\|<\operatorname{diam} \sigma\left(V_{n}\right) / 2$. This ensures that the unitary operator $U_{1} U_{2} \ldots U_{n-1} U_{n}=\left(U_{1} U_{2} \ldots U_{n-1}-\alpha^{n-1} I\right) U_{n}+\alpha^{n-1} U_{n}$ cannot be of the form $\lambda_{0} I+K_{0}$ for some $\lambda_{0} \in \mathbb{C}$ and some compact $K_{0}$ since otherwise we have (by taking the essential norm)

$\left\|\lambda_{0} I-\alpha^{n-1} U_{n}\right\|=\left\|\lambda_{0} I-\alpha^{n-1} U_{n}\right\|_{e s s} \leq\left\|U_{1} U_{2} \ldots U_{n-1}-\alpha^{n-1} I\right\|<\operatorname{diam} \sigma\left(U_{n}\right) / 2$

which is impossible. The same applies to $V_{1} V_{2} \ldots V_{n-1} N_{n}$ where $N_{n}=V_{n} \oplus K / r$. Hence, also $T_{1}=r^{n} U_{1} U_{2} \ldots U_{n-1} U_{n}$ and $T_{2}=r^{n} V_{1} V_{2} \ldots V_{n-1} N_{n}$ are not the sum of a scalar and a compact operator, and, therefore, $T=T_{1} \oplus T_{2}$ is a group commutator by Theorem 4 of [1]. Since $N=r U_{1} \oplus r U_{2} \oplus \ldots \oplus r U_{n} \oplus r V_{1} \oplus r V_{2} \oplus \ldots \oplus r N_{n}$, we see that $N$ is a product of three $n$-th roots by Lemma 1 .

To obtain the desired decomposition of $U$ take $\gamma \in \sigma(U), \gamma \neq \alpha$, and then choose $\beta \in \sigma(U)$, different from $\alpha$ and $\gamma$, such that $2 n|\alpha-\beta|<|\gamma-\beta|$ and such that $\alpha$ is the limit of a sequence of spectral points belonging to the open arc from $\alpha$ to $\beta$. (This can be done because $\alpha$ is not an isolated point in $\sigma(U)$.) Divide the closed arc from $\alpha$ to $\beta$ into $2(n-1)$ subarcs and the rest of the unit circle into two disjoint Borel subsets in such a way that each subset contains an infinite number of spectral points. Then use spectral projections to get $U_{1}, U_{2}, \ldots, U_{n}$ and $V_{1}, V_{2}, \ldots, V_{n}$ acting on infinite-dimensional subspaces. Adjust this partition, if necessary, to ensure $\beta, \gamma \in \sigma\left(U_{n}\right) \cap \sigma\left(V_{n}\right)$. In this case we have $|\gamma-\beta| \leq \operatorname{diam} \sigma\left(U_{n}\right)$ and $|\gamma-\beta| \leq \operatorname{diam} \sigma\left(V_{n}\right)$ which gives the desired estimates for the norms of $U_{i}-\alpha I$ and $V_{i}-\alpha I, i=1,2, \ldots, n-1$.

To resume, we have the following general theorem for normal operators:

Theorem 1. Every invertible normal operator $N$ not of the form $N=\alpha I+K$, $|\alpha| \neq 1$ and $K$ compact, on an infinite-dimensional Hilbert space is a product of three 
$n$-th roots for any $n>2$ and at least one of the factors is unitary. If $N=\alpha I+K$, $|\alpha| \neq 0,1$ and $K$ compact, then $N$ is a product of four $n$-th roots and two of them are unitary. In general this result is best possible.

Proof. The proof follows from Propositions 1, 2, 3, 4 and 5.

Corollary 2. An invertible normal operator $N$ is a group commutator if and only if for every $n>2$ the operator $N$ is a product of three $n$-th roots.

Proof. If $N$ is a group commutator, then by Theorem 5 of 1 it is not of the form $N=\alpha I+K$ with $|\alpha| \neq 1$ and $K$ compact. Hence, by Theorem 1 above $N$ is a product of three $n$-th roots for every $n>2$. Conversely, if $N$ is a product of three $n$-th roots for every $n>2$, this is true in particular for $n=3$, and by Proposition 4, $N$ is not of the form $N=\alpha I+K$ with $|\alpha| \neq 1$ and $K$ compact. Consequently, it is a group commutator by Theorem 5 of [1].

Remark 5. Corollary 2 gives a necessary and sufficient condition for a normal invertible operator to be a group commutator in terms of its factorization into three $n$-th roots for every $n>2$. In fact, it is enough to verify this condition for $n=3$ only. However, the question remains whether the operator $N=\alpha I+K$ with $|\alpha| \neq 0,1$ and normal compact $K$, hence a normal $N$ which is not a group commutator, can be written as a product of three $n$-th roots for an $n>3$ (see Remark 4). In particular, is $2 I=X Y Z$ for $X^{4}=Y^{4}=Z^{4}=I$ ?

Corollary 3. A general invertible operator $T$ on an infinite-dimensional Hilbert space is a product of five $n$-th roots for every $n>2$ and three of the factors can be chosen to be unitary.

Proof. By polar decomposition we have $T=P U$ where $P$ is positive and $U$ is unitary. By Theorem $1, P=K_{1} K_{2} V_{1} V_{2}$ with $K_{1}^{n}=I, K_{2}^{n}=I, V_{1}^{n}=I, V_{2}^{n}=I$ and $V_{1}, V_{2}$ unitary. Then $T=K_{1} K_{2} V_{1} V_{2} U=K_{1} K_{2} W$ and since $W=V_{1} V_{2} U$, being unitary, is also a product of three unitary $n$-th roots by Corollary 1 , we are done.

Again, the question is whether five factors are needed, or a general invertible operator $T$ can be written as a product of fewer than five $n$-th roots for an $n>2$.

\section{ACKNOWLEDGEMENT}

The authors are grateful to the referee for pointing out a mistake in the first proof of Proposition 5 and for other useful suggestions.

\section{REFERENCES}

[1] A. Brown, C. Pearcy, Multiplicative commutators of operators, Canad. J. Math. 18 (1966), 737-749. MR 34:608

[2] D.Ž. Djoković, Products of two involutions, Arch. Math. 18 (1967), 582-584. MR 36:2630

[3] L. Grunenfelder, T. Košir, M. Omladič, and H. Radjavi, On groups generated by elements of prime order, Geom. Dedicata 75 (1999), 317-332. CMP 99:14

[4] W.H. Gustafson, P.R. Halmos, and H. Radjavi, Products of involutions, Lin. Alg. Appl. 13 (1976), 157-162. MR 53:3135]

[5] P. Halmos, S. Kakutani, Products of symmetries, Bull. Amer. Math. Soc. 64 (1958), 77-78. MR 20:6658 
[6] H. Radjavi, The group generated by involutions, Proc. Royal Irish Acad. 81A (1981), 9-12. MR 83c: 47006

[7] M.J. Wonenburger, Transformations which are products of two involutions, J. Math. Mech. 16 (1966), 327-338. MR 34:5850

Department of Mathematics, University of Ljubljana, Jadranska 19, 1000 Ljubljana, SLOVENIA

E-mail address: milan.hladnik@fmf.uni-lj.si

Department of Mathematics, University of Ljubljana, Jadranska 19, 1000 Luubljana, SLOVENIA

E-mail address: matjaz.omladic@fmf.uni-lj.si

Department of Mathematics, Statistics and Computing Science, Dalhousie University, Halifax, Nova Scotia, Canada B3H 3J5

E-mail address: radjavi@mscs.dal.ca 